\title{
Echocardiography in the diagnosis left ventricular noncompaction
} Ze-Zhou Song

Address: Department of Ultrasound, The First Affiliated Hospital, College of Medicine, Zhejiang University, Hangzhou, PR China

Email: Ze-Zhou Song - zezhou_song@126.com

Published: 23 December 2008

Cardiovascular Ultrasound 2008, 6:64 doi:10.1 |86/1476-7|20-6-64

This article is available from: http://www.cardiovascularultrasound.com/content/6/1/64

(c) 2008 Song; licensee BioMed Central Ltd.

This is an Open Access article distributed under the terms of the Creative Commons Attribution License (http://creativecommons.org/licenses/by/2.0), which permits unrestricted use, distribution, and reproduction in any medium, provided the original work is properly cited.
Received: II November 2008

Accepted: 23 December 2008

\begin{abstract}
Echocardiography is the method of choice to establish a diagnosis and determine a treatment plan for patients with noncompaction of ventricular myocardium (NVM). The 2-dimentional echocardiography, 3-dimentional echocardiography, color Doppler echocardiography and contrast-enhanced echocardiography are of critical importance for diagnosis and family screening of NVM.
\end{abstract}

\section{Introduction}

In normal human hearts of children and adults the left ventricle (LV) has up to 3 prominent trabeculations and is, thus, less trabeculated than the right ventricle $[1,2]$. Rarely, more than 3 prominent trabeculations that is the so-called LV noncompaction of ventricular myocardium (NVM) can be found at autopsy and by various imaging techniques including echocardiography and MRI etc. in the LV.

NVM is recently included in the 2006 classsification of cardiomyopathies as a Genetic Cardiomyopathy [3]. NVM occurs because of a disorder of endomyocardial morphogenesis that results in a failure of trabecular compaction of the developing myocardium [4]. In adult patients one or more segments, especially the apical, mid-lateral and midinferior regions, of the left ventricle, and sometimes both ventricles, are characterized by numerous sinusoids or trabeculae that are excessive in number and abnormal in prominence and by deep intratrabecular recesses covered by endothelium that exhibits continuity with ventricular endocardium(Figure 1). Numerous modalities have been used in the description, characterization, and diagnosis of NVM including, but not limited to, magnetic resonance imaging, two-dimensional echocardiography (2DE), contrast-enhanced 2DE, and angiography $[5,6] .2 \mathrm{DE}$ is by far the most commonly used diagnostic modality. On the basis of echocardiographic studies, the prevalence of NVM has been estimated at $0.05 \%$ in the general population [7]. Therefore, the following review aims to give an overview about the current knowledge and controversial issues regarding echocardiography in the diagnosis and management of NVM.

\section{Diagnosis \\ Diagnostic criteria}

There are 2 sets of echocardiographic criteria for IVNC diagnosis: the Jenni criteria, which stress the presence of a 2-layered structure, and the Chin criteria, which focus on the depth of the recess compared with the height of the trabecula $[8,9]$. In both sets, it is important that there are no other cardiac structural abnormalities, such as semilunar valve obstruction or coronary artery anomalies. A comparison of these 2 sets of criteria is presented in Table $1[10]$.

\section{DE Diagnosis of NVM}

2DE is by far the most commonly used diagnostic modality. However, recent studies have found that the diagnosis of this disorder is often delayed because of difficulty in elucidating the diagnostic findings [11]. Frequently, multiple 2DE studies are required before the diagnosis is 


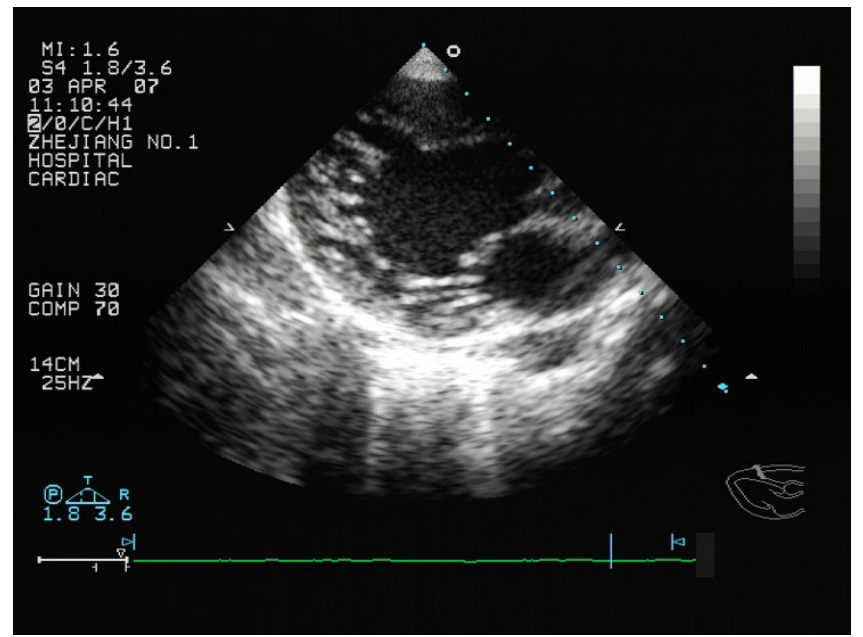

Figure I

left ventricular long axis view showing the trabeculationsof the left ventricular wall and deep intertrabecular recesses (arrow) by two-dimension echocardiograms.

made. 2DE provide excellent visualization of left ventricular cavity, but are not necessarily adequate for thorough inspection of myocardium and endocardium of entire left ventricular. The prominent trabeculations that characterize NVM may curve into left ventricular cavity in a manner that challenges the planar cuts that are inherent to 2DE. These limitations of 2DE may lead to misinterpretation of prominent trabeculations as "false tendons," and may also lead to underestimation of the severity of NVM. As a result, the 2DE diagnosis of NVM is often not made until patients had several 2DE. Ichida et al. [12] found that the diagnosis was missed altogether in well over half of their study population of children, and similarly, Baker et al. [13] report that three cases underwent an initial 2DE that did not confirm NVM which may be due to the nonuniform nature of NVM.

Table I: Echocardiographic Criteria for the Diagnosis of NVM

\section{Color Doppler echocardiography Diagnosis of NVM}

Occasionally, the affected myocardial segments are hypokinetic, contrary to certain forms of apical cardiomyopathy that can otherwise mimic NVM. However, differential echocardiographic diagnosis includes prominent normal myocardial trabeculations, false tendons and aberrant bands, cardiac tumors, and left ventricular apical thrombus. In these cases, the evidence of the direct blood flow from the ventricular cavity into deep intertrabecular recesses via color Doppler echocardiography analysis is helpful for the differentiation of NVM from other echocardiographic abnormalities in which this finding is not observed [8].

\section{Three-dimensional echocardiography (3DE) Diagnosis of NVM}

3DE allowed for accurate diagnosis, detailed characterization, and description of the extent of the affected myocardium in NVM in all four patients of this case series by Baker et al. [13]. Entire trabecular projections and intertrabecular recesses were visualized simultaneously and the distinction between the compact and noncompact LV myocardium was easily demarcated. Wall motion abnormalities were demonstrated simultaneously for large portions of myocardium. In contrast to $2 \mathrm{DE}, 3 \mathrm{DE}$ provides for pyramid-shaped datasets that encompass the entire left ventricular. Specifically, left ventricular can be sectioned in userselected planes and an unlimited number and angles of such planes can be used. Intracavitary echodensities that are suspicious for trabeculations can be tracked in multiple directions from base to apex. The resulting images provide the ability to visualize an entire trabeculation. The deep intertrabecular recesses and the distinction between the prominent noncompact left ventricular myocardium and the thin compact layer of left ventricular myocardium can also be well demonstrated [13].

\section{Contrast echocardiography Diagnosis of NVM}

Conventional echocardiography is the diagnostic modality of choice for INVM. In classic cases, the diagnosis of NVM is not difficult. However, when noncompaction is

\begin{tabular}{ll}
\hline Chin Criteria (1990) & Jennni Criteria (1999) \\
\hline $\begin{array}{ll}\text { Absence of any other coexisting cardiac structural abnormality } \\
\text { Numerous, excessively prominent trabeculations and deep }\end{array}$ & Absence of any other coexisting cardiac structural abnormality \\
$\begin{array}{l}\text { intertrabecular recesses } \\
\text { Views: parasternal long axis, subxyphoid, and apical }\end{array}$ & $\begin{array}{l}\text { Numerous, excessively prominent trabeculations and deep } \\
\text { intertrabecular recesses }\end{array}$ \\
$\begin{array}{l}\text { Focus on depth of recesses } \\
\text { Measured in end-diastole }\end{array}$ & Views: parasternal short axis, and apical \\
$\begin{array}{l}\text { Ratio of distance from the epicardial surface to the trough of the } \\
\text { trabecular recesses and distance from the epicardial surface to peak of } \\
\text { trabeculation } \leq 0.5\end{array}$ & $\begin{array}{l}\text { Measured in end-systole } \\
\text { intertrabecular recesses suplied by intraventricular blood on color }\end{array}$ \\
\end{tabular}


subtle or incomplete, disorders such as prominent normal myocardial trabeculations, hypertrophic cardiomyopathy, dilated cardiomyopathy, and left ventricularapical thrombus should be included in the differential diagnosis. Conventional echocardiography has some diagnostic limitations in obese patients or patients with lung disease who have poor acoustic windows.

Currently, echocardiography with various contrast agents is being used to enhance endocardial border delineation $[14,15]$. Koo et al. [16] performed transpulmonary contrast echocardiography with intravenous injection of PESDA (a sonicated mixture of perflourocarbon $[8 \mathrm{cc}], 5 \%$ albumin $[4 \mathrm{cc}]$, and 5\% dextrose water [8 cc]). Opacification of ventricular cavity with a contrast agent clearly defined the endocardial border, and the authors obtained better images with this method than with conventional transthoracic 2DE. Therefore, when conventional echocardiographic images are poor or diagnosis is uncertain, contrast echocardiography can be helpful. This procedure can be performed without further patient discomfort, and can save time and money. Apical swirling or nonuniform distribution of contrast may simulate endocardial trabeculae, especially in patients with low ejection fraction. Higher doses of contrast or the use of new imaging techniques, such as power Doppler, can be helpful in these situations $[17,18]$. Therefore, the abovementioned study should be performed in the future.

\section{Echocardiography in family screening of NVM}

The familial character of LVNC has been recognized and probably up to $44 \%$ of patients do have affected family members. Although there are no systematic investigations on the familial occurrence of LV NVM, there are single reports that demonstrate LV NVM to occur in multiple family members. Family screening may unmask affected family members for primary prevention including anticoagulation and implantable cardiovertor defibrillators (ICD) therapy, especially to early diagnosis of asymptomatic patients of NVM. 2DE, 3DE, Contrast echocardiography could all be the pretty good diagnostic modality of choice for NVM, which need to be further studied.

\section{Differential diagnosis of NVM}

The apical and midventricular segments of both the inferior and lateral wall are affected in more than $80 \%$ of patients $[8,19]$. Involvement of the midventricular anterior wall, basal segments, and septum is much less frequent [8]. The segmental appearance is an important feature, because prominent trabeculae may also be seen in hypertrophied hearts [10].

Although echocardiography has been the diagnostic test of choice for noncompaction, other modalities have been used for the diagnosis, including contrast ventriculogra- phy [20], computed tomography [20], and MRI [6]. MRI provides good correlation withecho for localization and extent of noncompaction and is useful in cases with poor echocardiographic image quality [21]. In addition, the demonstration of differences in MRI signal intensity in noncompacted myocardium may help identify substrate for potentially lethal arrhythmias [22]. Although the diagnostic value of cardiac MRI seems to increase in parallel with technical refinements, echocardiography is generally considered the gold standard for the diagnosis of NVM. In most cases, cardiac MRI offered no additional information compared with echocardiography except for the detection of a thrombus which was hidden in the spongelike myocardium of NVM [23]. Cardiac MRI guarantees more operator independency and is less affected by technical limitations than echocardiography, but there has been no large-scale study comparing the 2 modalities [23].

Occasionally, the affected myocardial segments are hypokinetic, contrary to certain forms of apical cardiomyopathy that can otherwise mimic IVNC. However, differential echocardiographic diagnosis includes prominent normal myocardial trabeculations, false tendons and aberrant bands, cardiac tumors, and left ventricular apical thrombus. In these cases, the evidence of the direct blood flow from the ventricular cavity into deep intertrabecular recesses via color Doppler analysis is helpful for the differentiation of IVNC from other echocardiographic abnormalities in which this finding is not observed $[8,23]$.

\section{Conclusion}

In conclusion, echocardiography is the method of choice to establish a diagnosis and determine a treatment plan for patients with NVM. Despite the widespread use of echocardiography, NVM is commonly misdiagnosed because of the lack of knowledge of this disorder, with a significant negative impact on the prognosis of these patients. Therefore, it is still important to make echocardiographers more familiar with this condition and its pathology.

\section{Competing interests}

The authors declare that they have no competing interests.

\section{References}

I. Boyd MT, Seward JB, Tajik AJ, Edwards WD: Frequency and location of prominent left ventricular trabeculations at autopsy in $\mathbf{4 7 4}$ normal human hearts: implications for evaluation of mural thrombi by two-dimensional echocardiography. J Am Coll Cardiol 1987, 9:323-326.

2. Sedmera D, Pexieder T, Vuillemin M, Thompson RP, Anderson RH: Developmental patterning of the myocardium. Anat Rec 2000, 258:319-337.

3. Maron BJ, Towbin JA, Thiene G, Antzelevich C, Corrado D, Arnett D, Moss AJ, Seidman CE, Young JB: AHA Scientific Statement. Contemporary Definition and Classification of the Cardiomyopathies. Circulation 2006, I 13:1807-1816. 
4. Weiford BC, Subbarao VD, Mulhern KM: Noncompaction of the ventricular myocardium. Circulation 2004, 109:2965-2971.

5. Lowery MH, Martel JA, Zambrano JP, Ferreira A, Eco L, Gallagher A: Noncompaction of the ventricular myocardium: The use of contrast-enhanced echocardiography in diagnosis. J Am Soc Echocardiogr 2003, 16:94-96.

6. Soler R, Rodriguez E, Monserrat L, Alvarez N: MRI of subendocardial perfusion deficits in isolated left ventricular noncompaction. J Comput Assist Tomogr 2002, 26:373-375.

7. Petersen SE, Selvanayagam JB, Wiesmann F, Robson MD, Francis JM, Anderson RH, Watkins H, Neubauer S: Left ventricular non-compaction. Insights from cardiovascular magnetic resonance imaging. J Am Coll Cardiol 2005, 46: I0I-I 05.

8. Jenni R, Oechnlin E, Schneider J, Jost CA, Kaufman PA: Echocardiographic and pathoanatomical characteristics of isolated left ventricular non-compaction: a step towards classification as a distinct cardiomyopathy. Heart 200I, 86:666-67I.

9. Chin TK, Perloff JK, Williams RG, Jue K, Mohrmann R: Isolated noncompaction of left ventricular myocardium: a study of eight cases. Circulation 1990, 82:507-5I3.

10. Božić I, Fabijanić D, Carević V, Polić S: Echocardiography in the diagnosis and management of isolated left ventricular noncompaction: case reports and review of the literature. J Clin Ultrasound 34:4I6-42I.

11. Toyono M, Kondo C, Nakajima Y, Nakazawa M, Momma K, Kusakabe $\mathrm{K}$ : Effects of carvedilol on left ventricular function, mass, and scintigraphic findings in isolated left ventricular non-compaction. Heart 200I, 86: E4.

12. Ichida F, Hamamichi Y, Miyawaki T, Ono Y, Kamiya T, Akagi T, Hamada H, Hirose O, Isobe T, Yamada K, Kurotobi S, Mito H, Miyake T, Murakami Y, Nishi T, Shinohara M, Seguchi M, Tashiro S, Tomimatsu $\mathrm{H}$ : Clinical features of isolated noncompaction of the ventricular myocardium. J Am Coll Cardiol 1999, 34:233-240.

13. Baker GH, Pereira NL, Hlavacek AM, Chessa K, Shirali G: Transthoracic Real-Time Three-Dimensional Echocardiography in the Diagnosis and Description of Noncompaction of Ventricular Myocardium. Echocardiography 2006, 23:490-494.

14. Main ML, Grayburn PA: Clinical applications of transpulmonary contrast echocardiography. Am Heart J 1999, 137: 144-I53.

15. Mulvagh SL, DeMaria AN, Feinstein SB, Burns PN, Kaul S, Miller JG, Monaghan M, Porter TR, Shaw LJ, Villanueva FS: Contrast echocardiography: Current and future applications. American Society of Echocardiography Task Force on Standards and Guidelines for the use of Ultrasonic Contrast in Echocardiography. I Am Soc Echocardiogr 2000, I3:33|-342.

16. Koo BK, Choi D, Ha JW, Kang SM, Chung N, Cho SY: Isolated Noncompaction of the Ventricular Myocardium: Contrast Echocardiographic Findings and Review of the Literature. Echocardiography 2002, 19:153-156.

17. Hayashi Y, Hamada M, Hiwad K: Characterization of left ventricular opaci.cation using sonicated serum albumin in patients with dilated cardiomyopathy and myocardial infarction. Jpn Circ J 1998, 62:91-96.

18. Bednarz JE, Spencer KT, Weinert L, Sugeng L, Mor-Avi V, Lang RM: Identification of cardiac masses and abnormal blood flow patterns with harmonic power-Doppler contrast echocardiography. J Am Soc Echcardiogr 1999, I 2:87|-875.

19. Oechslin E, Attenhofer Jost CH, Rojas JR, Kaufmann PA, Jenni R: Long-term follow-up of $\mathbf{3 4}$ adults with isolated left ventricular non-compaction: a distinct cardiomyopathy with poor prognosis. J Am Coll Cardiol 2000, 36:493-497.

20. Conces DJ Jr, Ryan T, Tarver RD: Noncompaction of ventricular myocardium: CT appearance. Am J Roentgenol |99|, 156:717-718.

21. Junga G, Kneifel S, Von Smekal A, Steinert H, Bauersfeld U: Myocardial ischemia in children with isolated ventricular non-compaction. Eur Heart J 1999, 20:910-916.

22. Daimon Y, Watanabe S, Takeda S, Hijikata Y, Komuro I: Two-layered appearance of noncompaction of the ventricular myocardium on magnetic resonance imaging. Circ J 2002, 66:619-621.

23. Hany TF, Jenni R, Debatin JF: MR appearance of isolated noncompaction of the left ventricle. J Magn Reson Imaging 1997, 7:437.

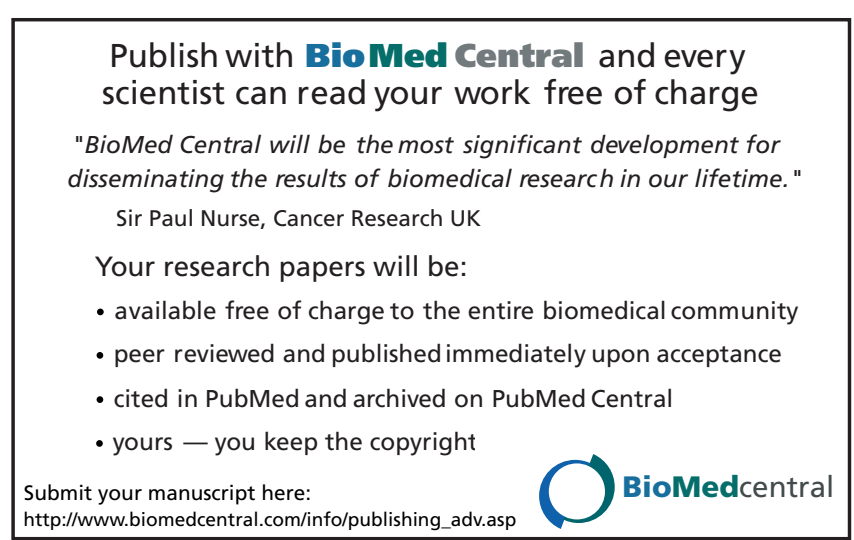

